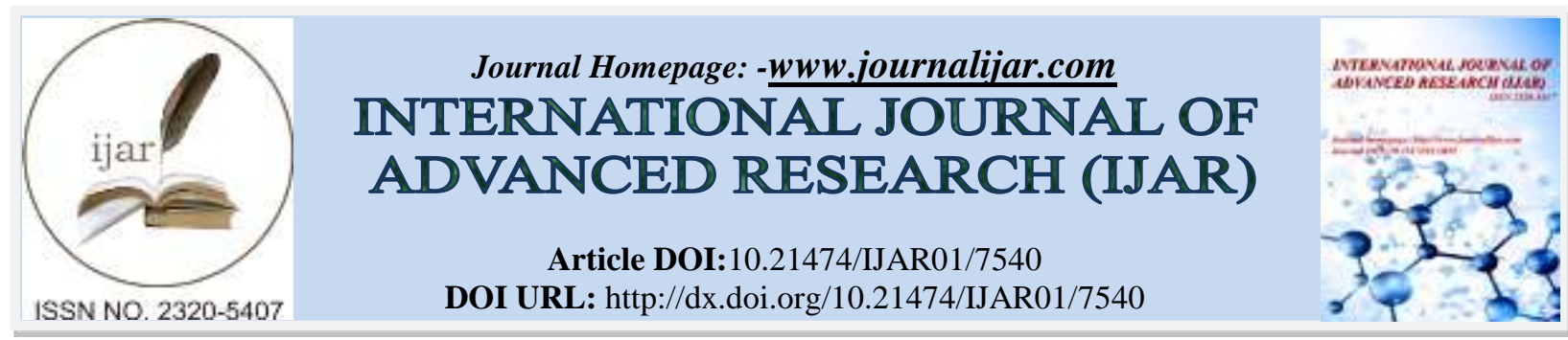

RESEARCH ARTICLE

\title{
PREVALENCE AND CHARACTERISTICS OF CERVICAL SPINE INJURY AMONG PATIENTS WITH TRAUMATIC BRAIN INJURY IN SAUDI ARABIA.
}

\author{
Alzahrani Abdullah $\mathbf{J}^{1}$, Aljabarti Azzah A ${ }^{1,2}$, Boker Faisal A ${ }^{1}$, Alshehri Mohammed $\mathbf{A}^{\mathbf{1}}$ and Sebeih Sami \\ $\mathbf{H}^{2}$.
}

1. College of Medicine, King Saud bin Abdulaziz University for Health Sciences, Saudi Arabia.

2. Emergency Medicine Department, King Abdulaziz Medical city, Jeddah, Saudi Arabia.

\section{Manuscript Info}

Manuscript History

Received: 11 June 2018

Final Accepted: 13 July 2018

Published: August 2018

Keywords:-

Cervical spine injury, Traumatic brain injury, cervical spine fracture, head injury.

\begin{abstract}
Traumatic Brain Injury (TBI) is commonly associated with other injuries, such as cervical spine injury (CSI), which critically affects TBI patients, interfering with airway management techniques, selection of proper radiological studies, and the management of both injuries. This study aimed to assess the prevalence, mechanism of injury, and characteristics of CSI in patients who sustain TBI. In addition, it aimed to identify the associated injuries in patients experiencing both TBI and CSI, and their effect on patient mortality and course of hospitalization. The study was conducted in the Health Information Management department. The charts of all adult (>14-year-old) patients presenting with TBI to the Emergency Department of King Abdulaziz Medical City in Jeddah or whom had been referred from other hospitals over a four-year period (January 2013 to December 2016), were reviewed. 220 patients with traumatic brain injury were included in the study. Most of the patients were young adults (median age 29 years). In 185 of the 220 patients (84.1\%), a diagnosis of CSI was ruled out on the basis of clinical presentation and computed tomography scan reports. A diagnosis of CSI was confirmed in the remaining 35 patients (prevalence $15.9 \%$ ). The prevalence of facial injury was higher among patients with CSI than among those without, as were the prevalence of thoracic trauma, rib fractures, thoracolumbar fractures, and abdominal injury. The prevalence of CSI was highest among young adults (greater in men than women) who had sustained motor vehicle-related injury, and CSI was usually associated with thoracic trauma, rib fractures and abdominal injury, that were consistent with the mechanism of injury.
\end{abstract}

Copy Right, IJAR, 2018,. All rights reserved.

\section{Introduction:-}

Traumatic brain injury (TBI) is a major public health problem, and is linked with significant morbidity and mortality in all age groups. TBI is defined as an insult to the brain caused by an external force, leading to temporary or permanent impairment of cognitive, physical, and psychosocial functions, with an associated alteration in the level of consciousness [1]. This type of trauma is commonly associated with the presence of other injuries, such as cervical spine injury (CSI), which critically affect TBI patients, interfering with airway management techniques, 
selection of proper radiological studies, and the management of both injuries [2]. The reported prevalence of CSI in patients with head trauma, regardless of age, ranges from 5-10\%, and rises with the severity of head injury, determined by using the Glasgow Coma Scale (GCS) [2,3]. A few risk factors have been identified for concurrent TBI and CSI, including the mechanism of injury, the characteristics and location of CSI, and the presence of other associated injuries [4,5]. More detailed characterization of such risk factors will be particularly useful for the early diagnosis and management of such trauma, which may be difficult to recognize due to an altered state of consciousness, and the overall severity of the trauma. Failure to detect an unstable CSI during the initial evaluation may worsen the neurological consequences, as previously reported [5]. This study aimed to assess the prevalence, mechanism of injury, and characteristics of CSI in patients who sustained TBI. It also aimed to identify any associated injuries in patients experiencing both TBI and CSI, and their effect on patient mortality and course of hospitalization.

\section{Methodology:-}

This was a cross-sectional study that aimed to determine the prevalence of CSI among patients of $>14$ years who were hospitalized because of TBI. The protocol was approved by the institutional review board of the host institution. The degree of TBI was assessed clinically using an immediate post-resuscitation Glasgow Coma Scale (GCS) score, and the presence of TBI was confirmed by computed tomography (CT) in all patients. All patients were managed using a multidisciplinary team approach and then admitted to a surgical ward at the same institution. The reason for hospital admission was typically multiple traumas, including the TBI. Patients were excluded from the study if they had a history of prior CSI.

The study was conducted in the Health Information Management (HIM) department. The charts of all patients of $>14$ years who presented to the Emergency Department (ED) at King Abdulaziz Medical City in Jeddah with TBI, or whom were referred from another hospital, over a four-year period (January 2013 to December 2016) were reviewed. All data were collected from patient records and the Centricity database for details of radiological imaging, with the help of the Hospital Information Coding Service. The criteria for the diagnosis of TBI were prespecified in the study protocol and were based on both clinical history and CT scan results. The information extracted included demographic data, the presence of TBI, the presence of other associated injuries, and the occurrence of complications during the patients' hospital stay. In patients who had sustained CSI, more detailed data regarding the type and location of the CSI were also collected.

Pre-admission management depends primarily on the severity of the head injury, the presence of other associated injuries, including CSI, and cardiopulmonary stability. All patients were maintained on a spinal backboard, a rigid cervical collar, and supportive blocks placed on both sides of the head, if CSI was suspected. At initial presentation to the ED the presence of an adequate airway and circulation were ensured. The presence or absence of CSI was determined using CT in the ED, as per hospital protocol. The scans were interpreted by qualified radiologists and the location and distribution of CSI were documented. All patients with moderate or severe TBI were managed medically in the intensive care unit (ICU) after stabilization, or after surgical intervention if indicated.

All patients who fulfilled the study criteria and presented to the ED during the study period were included in the study. The prevalence of CSI and associated injuries were calculated for the entire group of patients. To compare the baseline characteristics between patients with and without CSI, the Chi-square and Fisher's Exact tests were used for categorical variables, and Student's t-test was used for continuous variables. Odds ratios (ORs) and P-values were calculated, quoting 95\% confidence intervals. All analyses were performed using SPSS software, version 23.0 (SPSS).

\section{Results:-}

Prevalence of cervical spine injury:-

A total of 292 patients were admitted to the ED with TBI during the study period. Of these, 41 presented with an altered level of consciousness that was not traumatic in origin, and 31 did not have complete information in their records. Therefore, data from 220 patients with TBI were analyzed. The main demographic and clinical characteristics of the patients are provided in Table 1. Most of the patients were young adults (median age 29 years). In 185 of the 220 patients $(84.1 \%$ ), a diagnosis of CSI was ruled out on the basis of the clinical presentation and CT scan reports. In the remaining 35 patients, CSI was confirmed, giving a prevalence of $15.9 \%$. 


\section{CSI morphology and location in TBI patients:-}

Among the 35 patients in whom CSI was diagnosed by CT, the lower cervical vertebrae (C3-C7) were the most frequently injured, in $22(62.9 \%)$ patients, 19 of whom demonstrated a lower cervical injury alone, while the remaining three patients also had an upper cervical (C1-C2) injury. The most common type of CSI was a lateral mass fracture, which was present in $11(31.4 \%)$ patients, and more frequent in the upper segments (C1-C2). Transverse process fractures were also common, occurring in $10(28.6 \%)$ patients, and these tended to be in the lower segments (C3-C7). Transverse foramen fractures were documented in eight (22.9\%) patients, frequently in the lower segments (C3-C7), and a similar percentage of patients demonstrated an odontoid fracture, but this was usually located in the upper segments (C1-C2). Articular facet fractures were reported seven (20.0\%) times, always in the lower segments (C3-C7). Cervical spine fracture-subluxations were present in six (17.1\%) patients, half of which were associated with articular facet fracture, and there were no subluxations without fracture. Pedicle fractures were observed in four patients in the lower segments (C3-C7), and four (11.4\%) patients had lamina fractures, all of which were in the lower segments (C3-C7). In the lower segments (C3-C7), teardrop fractures were identified in three $(8.6 \%)$ patients, spinous process fractures in two $(5.7 \%)$ patients, and anterolisthesis in one (2.9\%) patient.

\section{Other associated injury with traumatic brain injury:-}

Other associated injuries were present in 150 of the 220 patients who had TBI $(68.2 \%)$. For all patients analyzed in the study the prevalence of the associated injuries are given in Table 1. The prevalence of facial injury was higher among patients with CSI than among those without, as were the prevalence of thoracic trauma, rib fractures, thoracolumbar fractures, and abdominal injury. Of the 35 patients with CSI, five (14.3\%) had no other associated injuries. The ORs for the presence of other associated injuries with CSI were not statistically significant for facial, upper limb, and lower limb fractures. CSI was significantly associated with higher risk of developing thoracic trauma, rib fractures, and abdominal injury. There was a significant difference in the length of hospitalization for patients who had sustained CSI compared to those who had not. There was no difference in the mortality of patients with and without CSI, but this was higher when the patients had several associated injuries. There were 17 deaths among all the patients, five of whom had sustained CSI. One patient who had a severe upper cervical injury stayed in the ICU for six days before dying. The other four patients had a CSI, accompanied by four or more other injuries, including abdominal injury in three patients.

\section{Discussion:-}

Data regarding the prevalence, demographics, severity of TBI, and mechanism of CSI, is useful to determine the best approach for high risk patients with TBI in the ED. Our study used medical records and CT scans of such patients to show a high prevalence of CSI in patients with TBI (15.9\%, or $~ 1$ in 6 patients). Perhaps unsurprisingly, young males with motor vehicle-related injury were more likely to have CSI, as were those with severe head injury. However, the proportion of patients who had sustained CSI that was not associated with these factors was not negligible.

The high prevalence of CSI in our TBI cohort is inconsistent with those reported elsewhere [5,6,7]. However, it should be noted that these recent studies did not account for the differences between children and adults, including the clinically distinct type of cervical spine and skull injuries that result from trauma in children. Also, these studies did not include patients who sustained mild TBI, due to a low energy impact, which may have resulted in an underestimation of the prevalence of CSI. In contrast, our study was only of adult patients, all of whom underwent CT for the diagnosis of CSI, regardless of the severity of TBI that was suggested clinically.

Our study reported a high proportion of CSIs occurring in young adults, but there was no statically significant difference from elderly patients. Unexpectedly, a previous study found that older patients had a significantly higher risk of developing CSI following a motor vehicle accident, but there was no difference in the prevalence of CSI following falls between age groups [7]. This may be explained by differences in the effects of high-energy trauma, and by the presence of age-associated cervical spondylosis, which predisposes elderly patients to CSI. Although the severity of TBI is proportionally related to the presence of CSI, the GCS score should not be used as a predictor of concomitant CSI because it can be affected by other factors, for example the use of alcohol or sedatives, and/or intubation. 
Several previous studies have shown an overall interaction between CSI and injury mechanism, and found that patients with vehicular-related head trauma were significantly more likely to have sustained CSI than those who suffered non-vehicular-related trauma [5,8]. This can be attributed to the excessive force directed at the skull and then transmitted to the cervical spine, or which affects the cervical spine directly. In particularly, when a safety belt is not fastened, the passenger is susceptible to secondary cervical spine trauma, because the neck is easily hyperflexed or hyperextended by a collision with a structure inside the vehicle, such as the windshield or steering wheel $[9,10]$.

An association between CSI and TBI in patients sustaining head injury would be anticipated because of the close anatomical proximity of the structures. Unexpectedly, however, we found that the lower segments (C3-C7) were more likely to be affected by trauma than the upper segments (C1-C2) of the cervical spine. However, these findings are not consistent with those of previous studies, which suggested that upper CSI was more likely $[5,6,8]$. Anatomically, the articulating margins of the atlas and axis are more horizontal in orientation than in the remainder of the cervical spine, because the axis forms the pivot upon which the atlas rotates. These features allow a wide range of rotatory motion, but render $\mathrm{C} 1-\mathrm{C} 2$ susceptible to injury by certain traumatic forces [11]. This is because any axial force tends to impose stress upon the weakest point of the atlas, at the junction of the anterior arch and the lateral mass. This may explain why injuries to the lateral mass were common in the upper segments in our study.

When other injuries occur alongside TBI, higher morbidity, greater impairment of quality of life, and longer periods of hospitalization result. Hence, our secondary objective was to characterize individuals with multiple such injuries and to investigate the possible risk factors. The Abbreviated Injury Scale (AIS) and the Injury Severity Score (ISS) are usually used to rate the severity of injury in patients with multiple trauma. The AIS method considers six anatomic regions of injury: the head/neck, face, thorax, abdomen, extremities, and external soft tissue [12,13]. ISS, which is determined by rating each injury using the AIS, has been shown to correlate significantly with mortality in the most severely injured patients [14]. Unfortunately, AIS and ISS had not been documented for the patients in this study. However, we categorized the other associated injuries into eight groups, detailed in Table 1, based on CT findings. An association between facial injury and CSI was expected, but was not statically significant in our cohort, as for upper and lower extremity fractures. However, rib fractures, thoracic trauma, and abdominal injury were associated with a significantly higher chance of CSI. This is consistent with the idea that mechanism of injury is the most important predictor of CSI in patients with TBI.

A few limitations to our study needed to be documented. First, data were collected from the Medical Records Department without randomization. Second, patients were included in the study if they were admitted to hospital after being diagnosed with TBI in the ED. As a result, this study did not include patients who visited the ED, but for whom hospitalization was not considered necessary. Third, several patients were referred from other local hospitals to our center because of the severity of their injuries, which may have contributed to the overall high prevalence and rate of complications. Fourth, Crashworthy systems and devices also affect injury risk in trauma patients. Therefore, adjusting for this confounding factor might affect the association between TBI and CSI. Finally, we did not collect information regarding management decisions and patient follow-up after the diagnosis of CSI, because this was not a study objective.

\section{Conclusion:-}

In conclusion, among adult patients who were hospitalized for TBI, concurrent CSI was confirmed in $15.9 \%$, usually in the lower (C3-C7) cervical vertebrae. The prevalence of CSI was highest in young adults (men greater than women) who sustained motor vehicle-related injury, and CSI was usually associated with thoracic, rib fractures, and abdominal injuries, which could only be explained by this mechanism of injury. Complete and rapid cervical spine evaluation is required in these patients. In this regard, investment in raising awareness of the importance of traffic laws and the use of safety equipment are vitally important.

\section{Acknowledgement:-}

We gratefully acknowledge the editorial advice and information received from Dr. Muhammad Anwar Khan from the Medical Education Department, Ahmed Aljuffri from the College of Medicine, whose comments greatly improved the manuscript. We are also immensely grateful to Mr. Ghazie Gebrihiwot from the Health Information Management Department, for assistance with coding services and patient information. 
Table 1:-Demographics and injury characteristics in 220 patients with traumatic brain injury:

\begin{tabular}{|c|c|c|c|c|c|}
\hline Characteristics & $\begin{array}{l}\text { All TBI } \\
\text { Patients } \\
(\mathbf{N}=\mathbf{2 2 0})\end{array}$ & $\begin{array}{l}\text { CSI } \\
\text { Confirmed } \\
(\mathbf{N}=\mathbf{3 5})\end{array}$ & $\begin{array}{l}\text { CSI } \\
\text { Ruled Out } \\
(\mathrm{N}=\mathbf{1 8 5})\end{array}$ & $\begin{array}{l}\text { Odds } \\
\text { Ratio } \\
(95 \% \\
\text { CI) } \\
\end{array}$ & P Value \\
\hline \multicolumn{6}{|l|}{ Gender } \\
\hline Male sex - no. $(\%)$ & $194(88.2 \%)$ & $29(82.9 \%)$ & 165 & 0.586 & $0.212 *$ \\
\hline Female sex - no. $(\%)$ & $26(11.8 \%)$ & $6(17.1 \%)$ & 20 & 1.707 & $0.212 *$ \\
\hline \multicolumn{6}{|l|}{ Age (in years) } \\
\hline Median (interquartile range) - yr. & $29(23)$ & $32(24)$ & $28(23)$ & & \\
\hline \multicolumn{6}{|l|}{ Level of TBI } \\
\hline Mild (GCS: 13-15) & $94(42.7 \%)$ & $11(31.43 \%)$, & $83(44.9 \%)$ & 0.563 & 0.141 \\
\hline Moderate (GCS: 9-12) & $32(14.5 \%)$ & $4(11.43 \%)$ & $28(15.1 \%)$ & 0.724 & 0.568 \\
\hline Severe (GCS: 3-8) & $94(42.7 \%)$ & $20(57.14 \%)$ & $74(40 \%)$ & 2.000 & 0.060 \\
\hline \multicolumn{6}{|l|}{ Mechanism of injury } \\
\hline Motor vehicle accident & $170(77.2 \%)$ & $35(100 \%)$ & $135(73 \%)$ & 1.259 & 0.001 \\
\hline Motorcycle accident & $7(3.2 \%)$ & & $7(3.7 \%)$ & & \\
\hline Fall & $29(13.2 \%)$ & & $29(15.7 \%)$ & & \\
\hline Car/pedestrian accident & $7(3.2 \%)$ & & $7(3.7 \%)$ & & \\
\hline Physical assaults & $3(1.4 \%)$ & & $3(1.6 \%)$ & & \\
\hline Unclassified direct trauma & $4(1.8 \%)$ & & $4(2.2 \%)$ & & \\
\hline \multicolumn{6}{|l|}{ Associated injuries } \\
\hline Facial trauma & $65(29.6 \%)$ & $14(40 \%)$ & $51(27.6 \%)$ & 1.752 & 0.139 \\
\hline Injuries to upper limbs & $52(23.6 \%)$ & $9(25.7 \%)$ & $43(23.2 \%)$ & 1.143 & 0.752 \\
\hline Thoracic trauma & $58(26.4 \%)$ & $14(40 \%)$ & $44(23.9 \%)$ & 2.136 & 0.046 \\
\hline Fractures of lower limbs & $30(13.6 \%)$ & $5(14.28 \%)$ & $25(13.5 \%)$ & 1.067 & $0.540 *$ \\
\hline Hip fracture & $20(9.1 \%)$ & $5(14.28 \%)$ & $15(8.1 \%)$ & 1.889 & $0.194 *$ \\
\hline Rib fractures & $35(15.9 \%)$ & $14(40 \%)$ & $21(11.4 \%)$ & 5.2 & 0.001 \\
\hline Thoracolumbar fractures & $38(17.3 \%)$ & $7(20 \%)$ & $31(5.9 \%)$ & 1.242 & 0.642 \\
\hline Abdominal injury & $7(3.2 \%)$ & $4(11.4 \%)$ & $3(1.6 \%)$ & 7.828 & $0.013 *$ \\
\hline \multicolumn{6}{|l|}{ Complications } \\
\hline Pneumonia & $8(3.64 \%)$ & $2(5.71 \%)$ & $6(3.2 \%)$ & 1.808 & $0.372 *$ \\
\hline Urinary tract infection & $3(1.37 \%)$ & & $3(1.6)$ & & \\
\hline Sepsis & $3(1.37 \%)$ & $1(2.86 \%)$ & $2(1.1 \%)$ & 2.691 & $0.407 *$ \\
\hline Respiratory failure & $2(0.91 \%)$ & $1(2.86 \%)$ & $1(0.5 \%)$ & 5.412 & $0.293 *$ \\
\hline $\mathrm{DVT} / \mathrm{PE}$ & $9(4.09 \%)$ & $2(5.71 \%)$ & $7(3.8 \%)$ & 1.541 & $0.434 *$ \\
\hline Seizure & $4(1.81 \%)$ & & $4(2.2 \%)$ & & \\
\hline \multicolumn{6}{|l|}{ Hospital course } \\
\hline Mean — days & 32 & 69 & 24 & & \\
\hline Need for ICU admission - no. (\%) & $135(61.4 \%)$ & $30(85.7 \%)$ & $105(56.8 \%)$ & 4.571 & 0.001 \\
\hline Deaths - no. $(\%)$ & $17(7.7 \%)$ & $5(2.3 \%)$ & $12(6.4 \%)$ & 2.403 & $0.111 *$ \\
\hline
\end{tabular}

*P values in these cohorts were calculated using Fisher's Exact Test. 


\section{References:-}

1. Faul M, Xu L, Wald MM, Coronado VG. Traumatic Brain Injury in the United States: Emergency Department Visits, Hospitalizations and Deaths, 2002-2006. Atlanta: Centers for Disease Control and Prevention, National Center for Injury Prevention and Control; 2010.

2. Hills MM, Deanne SA. Head injury and facial injury: is there an increased risk of cervical spine injury? J Trauma 1993; 34:549-54.

3. Michael DB, Guyot DR, Darmody WR. Coincidence of head and cervical spine injury.J Neurotrauma 1989; 6:177-89.

4. Sinclair D, Schwartz M, Gruss J, McLellan B. A retrospective review of the relationship between facial fractures, head injuries and cervical spine injuries. J Emerg Med 1988; 6:109.

5. Holly, Langston T, et al. Cervical spine trauma associated with moderate and severe head injury: incidence, risk factors, and injury characteristics. Journal of Neurosurgery: Spine 2002; 96.3: 285-91.

6. Iida H, Tachibana S, Kitahara T, et al. Association of head trauma with cervical spine injury, spinal cord injury, or both. J Trauma.1999; 46:450-52.

7. Fujii, Tomoko, Mark Faul, and Scott Sasser. Risk factors for cervical spine injury among patients with traumatic brain injury. Journal of emergencies, trauma, and shock 2013; 6.4: 252.

8. Bucholz RW, Burkhead WZ, Graham W, et al. Occult cervical spine injuries in fatal traffic accidents.J Trauma $1979 ; 19: 768-91$.

9. Tator $\mathrm{CH}$. Classification of spinal cord injury based on neurological presentation, in Narayan RK, Wilberger JE, Povlishock JT (eds): Neurotrauma. New York: McGraw-Hill, 1996, pp1059-72.

10. Greve MW, Zink BJ: Pathophysiology of traumatic brain injury. Mt Sinai J Med 2009; 76:97-104.

11. Bullock R, Chesnut RM, Clifton G, et al. Guidelines for the management of severe head injury. Eur J Emerg Med.1996; 3:109-27.

12. Greenspan L, McLellan BA, Greig H. Abbreviated injury scale and injury severity score: a scoring chart. J Trauma 1985, 25:60-4.

13. Chu, Dachen, et al. Prevalence of associated injuries of spinal trauma and their effect on medical utilization among hospitalized adult subjects-a nationwide data-based study. BMC health services research 2009; 9.1:137.

14. Baker SP, O'neill B. The injury severity score: an update. J Trauma 1976; 16:882-5. 